\title{
Statistical analysis of the mid-latitude trough position during different categories of magnetic storms and different storm intensities
}

Na Yang ${ }^{1,2,3}$, Huijun Le $e^{1,2^{*}}$ and Libo Liu ${ }^{1,2}$

\begin{abstract}
The ionospheric mid-latitude trough minimum position as a function of geomagnetic storm time is identified and investigated statistically in terms of the category and the intensity of storms. The data of ion density derived from DMSP and DEMETER satellites were used to extract the trough position. The variations of mid-latitude trough in 41 moderate magnetic storms and 88 intense magnetic storms in the 23rd solar cycle were studied. The results show that the trough moves toward the equator as Dst index decreases and toward the pole as Dst index increases. Compared with the ICME, MC and CIR storms, in sheath storms the trough shifts to lower latitude at the end of the main phase, although the average storm intensity is weak. During the storm recovery phase, the rapid recovery of the trough position can be seen at the start of the recovery phase for moderate CIR storms. We also calculated the correlation between the minimum latitude of the trough position and the storm magnitude as well as other related main phase parameters during all storms. We found that the minimum latitude of the trough position exhibits a strong correlation with the storm magnitude during magnetic storms. However, the correlation coefficients between the trough position and other related main phase parameters are very low.
\end{abstract}

Keywords: Ionosphere, Mid-latitude trough, Statistic analysis, Sheath storms, CIR storms, Storm intensity

\section{Introduction}

The mid-latitude trough is a typical feature of the nightside $\mathrm{F}$ region and lies at the interface between the midlatitude and auroral ionospheres. Throughout the current paper, 'trough' refers specifically to the mid-latitude trough. The trough has been studied for several decades (Muldrew 1965; Horvath and Essex 2003; Nilsson et al. 2005; Pryse et al. 2006; Middleton et al. 2008; He et al. 2011; Lee et al. 2011; Ishida et al. 2014). Most studies have displayed the quiet-time features of the trough (e.g., Rodger et al. 1992; Scali 1992; Rodger 2008). Some studies investigate the dynamics of the trough during substorms (e.g., Rodger et al. 1986; Rodger and Dudeney

\footnotetext{
${ }^{*}$ Correspondence: lehj@mail.iggcas.ac.cn

1 Key Laboratory of Earth and Planetary Physics, Institute of Geology

and Geophysics, Chinese Academy of Sciences, Beijing, China

Full list of author information is available at the end of the article
}

1987; Zou et al. 2011). Only a few studies focus on the trough dynamics during magnetic storms. These studies mainly studied the relationship between trough position and Kp index and Dst index for all phases of storms (e.g., Deminov et al. 1995a, b, 1996; Karpachev et al. 1996). However, the trough dynamics have not been investigated in the catalog of different magnetic storms.

In the near-Earth space, magnetic storms are predominantly driven by two different types of solar disturbances: coronal mass ejections (CMEs) and corotating interaction regions (CIRs). A CIR is formed at the interface between the high-speed solar wind and preceding low-speed solar wind. The high-speed solar wind originates from the regions of open magnetic field in the solar corona (Krieger et al. 1973). When a single coronal hole persists for many solar rotation periods, CIRs may recur with a $\sim 27$-day period (or more frequently if more than one coronal hole is presented on the sun) (Tsurutani et al. 
2006). CMEs with vast explosions of plasma and embedded magnetic flux erupt from magnetic fields associated with sunspots (Webb and Howard 2012). Interplanetary coronal mass ejections (ICMEs) are generally believed to be the counterparts of CMEs at the sun (Richardson and Cane 2010). ICMEs occur predominantly during solar maximum and generally cause the largest excursions in the Dst and Kp indices (Richardson and Cane 2012). ICME storms are further classified as either magnetic clouds (MC) storms or sheath storms by the geoeffective structure. Magnetic clouds are a subset of CME ejecta. Sheath regions are ahead of the CME ejecta. Magnetic clouds are identified in the solar wind by a strong magnetic field with a large rotation of the magnetic field direction (Burlaga et al. 1981; Klein and Burlaga 1982). In the sheath regions, the solar wind plasma is compressed and heated.

Several studies have contrasted the effects of ICMEs and CIRs (Wilken et al. 1999; Dmitriev et al. 2005). Huttunen et al. (2002) found that sheath regions drove stronger auroral activity, while magnetic clouds drove stronger ring current enhancement. Borovsky and Denton (2006) showed 21 differences between ICME and CIR storms in a number of regions, including the solar wind, the magnetosphere and the auroral zone. Denton et al. (2006) compared average plasma sheet parameters for the two types of events. They found that CIR storms had a more important modulation in the plasma sheet temperature, while ICME storms had a more important modulation in the plasma sheet density. Katus et al. (2015b) showed that the responses of the plasmapause location were also very different.

The aim of the present study was to systematically examine the general features of the trough position by undertaking a superposed epoch analysis. We focus on the differences seen in different categories of magnetic storms and different storm intensities. The paper begins with reviewing satellite data, identification of storms and method of superposed epoch analysis in "Data and Methodology" section. In "Results" section, we present the results for different categories of magnetic storms and different storm intensities. Fourth and fifth sections present the "Discussion" and "Conclusions" of the paper.

\section{Data and methodology}

\section{Observations}

We examined nocturnal latitudinal profiles of the total ion density in 1996-2005 measured by the Special Sensor-Ions, Electrons, Scintillation (SSIES) instrument package onboard the Defense Meteorological Satellite Program's (DMSP) F12, F14 and F15 satellites. The DMSP satellites are in Sun-synchronous circular polar orbits at about $850 \mathrm{~km}$ altitude. The orbits of these DMSP satellites are on the 2100 LT meridian when in the nightside sector. In addition, we also examined nocturnal latitudinal profiles of the total ion density in 2005-2009 measured by the Plasma Analysis Instrument (IAP) onboard the DEMETER satellite. The French microsatellite DEMETER was launched in June 2004, and it was operated until December 2010 . The spacecraft had a nearly Sun-synchronous circular orbit $(\mathrm{LT} \approx 10.30$ and $\approx 22.30)$ at an altitude of about $700 \mathrm{~km}$.

\section{Selection of trough-like phenomena}

The trough minimum position can be found by analyzing latitudinal profiles of ion density between $40^{\circ}$ and $70^{\circ}$ magnetic latitude during each storm. The latitudinal range between $40^{\circ}$ and $70^{\circ}$ magnetic latitude has been demonstrated to be suitable by Yang et al. (2015). Deminov et al. (1995a) demonstrated that the trough position primarily depends on the actual magnetic activity level. So, the variation in the trough position during each storm is inspected visually. If the variation in the trough position is not consistent with the variation in the Dst index, corresponding trough signatures are removed. When the DMSP and DEMETER satellites are placed on the 2100 and 2230 LT meridians in the nightside sector, then the local time of the trough position is in the premidnight region. Therefore, the local time distribution of the trough position is small and the local time effect of the trough position can be neglected.

\section{Identification of storms}

Zhang et al. (2007a, b) classified 88 intense storms (Dst$\min \leq-100 \mathrm{nT})$ in the 23rd solar cycle (1996-2005) according to their solar wind driver. These 88 intense storms include 11 CIR storms and 77 ICME storms, of which 33 storms are driven by MC and 19 storms by sheath.

In order to further examine the response of trough position to storm intensity, we applied the following selection criteria. The moderate ICME storms $(-100 \mathrm{nT} \leq$ Dstmin $\leq-50 \mathrm{nT})$ studied in this study are selected on the basis of an initial ICME list (19962009) of Richardson and Cane (2010). The moderate CIR storms $(-100 \mathrm{nT} \leq$ Dstmin $\leq-50 \mathrm{nT})$ studied in this study are selected on the basis of an initial CIR list (1995-2004) of Jian et al. (2006). The CIR list (including 2005-2009) is later updated at http://www.srl.caltech. edu/ACE/ASC/DATA/level3/index.html.

The Dst index is then examined during each storm. Selection of moderate storms began with a broad search for all storms with the peak Dst between -50 and $-100 \mathrm{nT}$. According to the selection criteria provided by Denton et al. (2006), storms are rejected when either (1) one or more events occur within the $72 \mathrm{~h}$ after the initial 
event, or (2) clear minimum storm Dst is not found during each event occurred. These criteria provide a list of 16 CIR and 25 ICME moderate storms. It should be noted that these moderate ICME storms contain MC storms due to the nature of the initial ICME list. Therefore, the storms studied in "Superposed epoch analysis results for CIR storms and ICME storms" and "Superposed epoch analysis results for moderate storms and intense storms" sections contain intense MC storms.

Figure 1 shows the distribution of ICME and CIR storms as well as the corresponding minimum Dst index for each event. It is clear that the majority of intense storms are ICME storms, and the moderate storms show a significant proportion of CIR storms. During solar maximum, ICME storms are typically most numerous; in contrast, CIR storms are sparse.

\section{Superposed epoch analysis}

In this study, a superposed epoch analysis similar in method to other recent studies (Katus et al. 2013, 2015a, b) is employed. This method uses multiple markers in each storm to normalize the time line. First, the time of the storm peak Dst is defined as the end of the main phase. The end of the recovery phase is then defined as the maximum Dst in the following $96 \mathrm{~h}$. The start of the main phase is selected as the maximum Dst in the $24 \mathrm{~h}$ prior to the storm peak. In this study, the identification of a storm sudden commencement is not required. The start of the initial phase is defined as the largest rate of Dst increase in the $8 \mathrm{~h}$ prior to the start of the

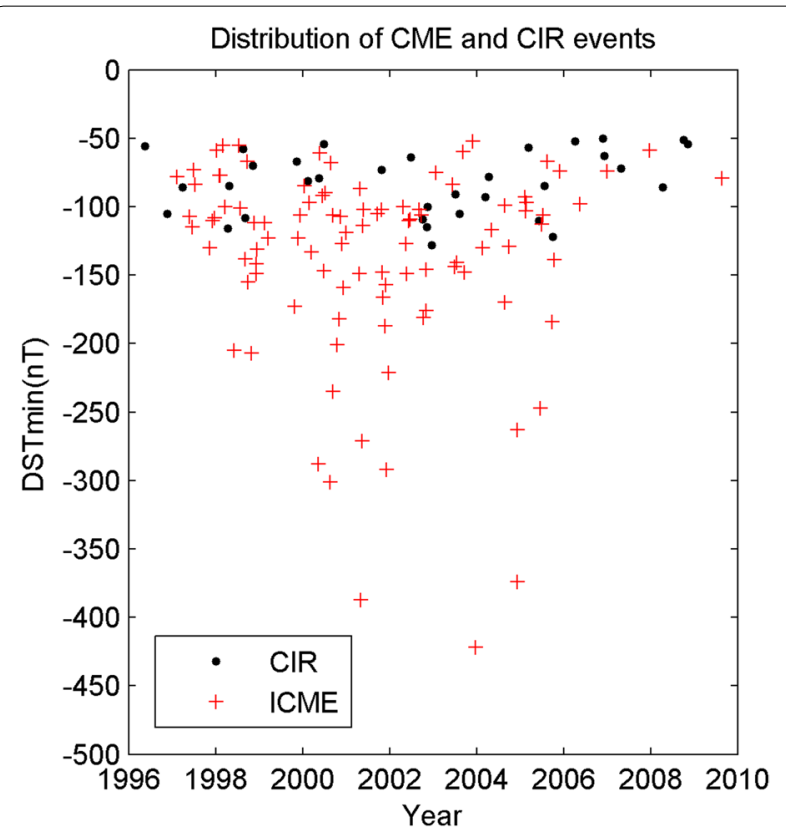

Fig. 1 Distribution of ICME storms and CIR storms used in this study main phase. Finally, the 6-h period before the start of the initial phase is concatenated to present information concerning the initial state of the magnetosphere. The epoch markers are then used to calculate the duration of each storm phase. These values are then utilized to normalize the epoch time line. Each storm phase is essentially stretched or compressed to the average duration of each phase using linear interpolation. The superposition used the 1-h time-resolution bins in the normalized time period. The process is repeated for trough positions.

\section{Correlation coefficient and $P$ value}

The correlation coefficients and $P$ values are calculated to determine the relationship between the minimum latitude of the trough position and the storm magnitude as well as other related main phase parameters during all storms. It is known that the confidence in a relationship is formally determined not just by the correlation coefficient but also by the number of pairs in the data. The number of pairs determines the 'significance' of the statistical analyses. The $P$ value is widely used to determine statistical significance in statistical hypothesis testing, specifically in null hypothesis significance testing. The $P$ value is defined as the probability of getting a correlation as large as the observed value by random chance, when the null hypothesis is true. A small $P$ value (typically $<0.05)$ is taken as strong evidence against the null hypothesis, then the null hypothesis can be rejected, and it can be concluded that the correlation $R$ is significant. $P$ values are usually found on a reference table by first calculating a Chi-square value.

\section{Results}

\section{Storm example}

The storm event on May 8, 2005, was associated with the passage of a CIR. Figure 2 describes time series of the Dst index and the Kp index, as well as the trough position derived from different satellites for this storm. The red vertical lines show the start or end of each phase. The red circles represent variations of trough position with longitude between 0 and 200. The black circles represent variations of trough position with longitude between 200 and 360. There are little black circles in Fig. 2d, which is because DMSP ion density data over this longitude range don't cover the trough region in the Southern Hemisphere. It is clear that the trough position depends on the current magnetic activity level. The trough moves toward the equator as Dst index decreases and toward the pole as Dst index increases. The behavior of the trough position mirrors that of the Dst index. However, it should be pointed out that the trough position reaches its minimum value in a few to several hours prior to the 

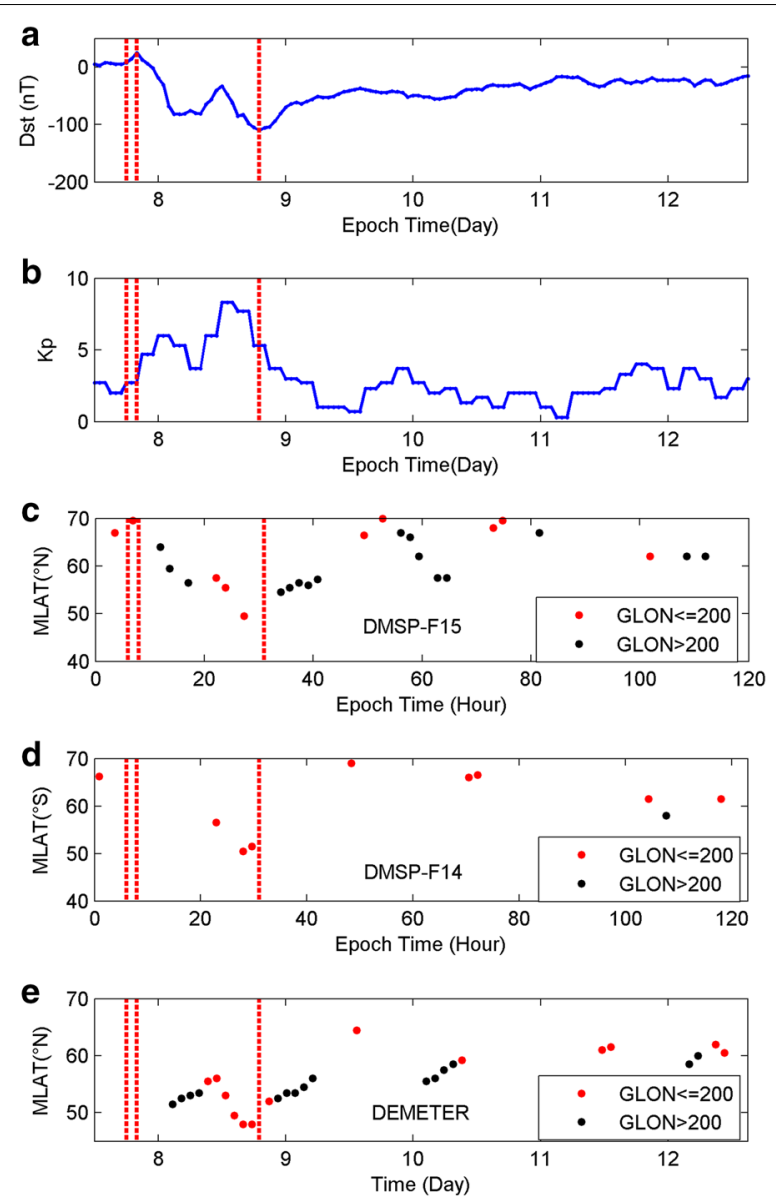

Fig. 2 May 8, 2005, CIR storm. a Dst index, b Kp index, c Northern Hemisphere trough positions derived from DMSP-F15 satellite data, d Southern Hemisphere trough positions derived from DMSP-F14 satellite data, e Northern Hemisphere trough positions derived from DEMETER satellite data. The red vertical lines show the start or end of each phase

minimum in Dst index, and the changes in Kp index are ahead of variations in trough position (see Fig. 2e). The results are consistent with the findings of Deminov et al. (1995a). During the storm recovery phase, the trough position recovers to pre-event values in about $20 \mathrm{~h}$ (see Fig. 2c). In general, the recovery of plasmapause has a larger timescale (Rodger. 2008). Therefore, the relationship between the trough and plasmapause breaks down during the storm recovery phase. It can be seen from Fig. $2 \mathrm{c}$ that the variations in the Dst index during the recovery phase of storm can result in large variations in the trough position. In addition, it is clear that the longitudinal effect is weak and can be neglected during the magnetic storm. This result is consistent with the finding of Karpachev et al. (1996). So, in the later study, the longitudinal effect of trough is neglected.

\section{Variations in the trough position of hemispheres}

To reveal the differences between the Northern and Southern Hemispheres in trough's response to magnetic storm, Fig. 3 shows the result for all of the MC storms because of their largest number of events. Figure 3a displays the Dst index data density as a function of epoch time. The color of the plot presents the density of data points in each bin. Each bin is $3 \mathrm{~h}$ across the $\mathrm{x}$ axis and $5 \mathrm{nT}$ across the y axis. The black and white lines indicate the mean and the median, respectively. Figure $3 \mathrm{~b}$ shows the trough positions as a function of epoch time in the Northern Hemisphere. The pink, green and black circles indicate the data derived from DMSP-F12, DMSP-F14 and DMSP-F15, respectively. The black and blue lines indicate the mean and the median, respectively. For clarity, Fig. $3 \mathrm{c}$ only shows the average trough positions and the upper and lower quartiles in both hemispheres along the normalized epoch time line. The black and blue lines indicate the results of the Northern Hemisphere and Southern Hemisphere, respectively. The red vertical lines show the start or end of each phase. As shown in Fig. 3a, the average Dst index increases with time during the initial phase, then drops to approximately $-130 \mathrm{nT}$ at the storm peak and then slowly recovers to initial value. Figure $3 \mathrm{~b}$ shows that the average trough position peaks at the peak of Dst index. It is striking that there is almost the same temporal evolution of the trough position in the Northern Hemisphere and Southern Hemisphere (see Fig. 3c). In quiet conditions, the difference between the trough positions of the Northern Hemisphere and that of the Southern Hemisphere is mainly due to the longitudinal difference. During magnetic storms, the longitudinal effect of the trough position can be neglected. Therefore, the trough position follows a similar variation throughout the storm sequence regardless of hemispheres. The result is also correct for the other three categories of magnetic storms. In the later text, we only present the results of the Northern Hemisphere.

\section{Superposed epoch analysis results for CIR storms and ICME storms}

Figure 4 shows the average Dst index and trough position for CIR (a) and ICME (b) storms with the peak Dst between -50 and $-150 \mathrm{nT}$, as a function of epoch time. The numbers of CIR and ICME storms are 27 and 60, respectively. It is clear that ICME storms cause larger excursions in the Dst index and trough position. During the storm main phase, changes in the trough position follow Dst index variations irrespective of storm drivers. During the storm recovery phase, the rapid recovery of the trough position can be found at the start of the CIR storm recovery phase, and the rate of recovery slows markedly with time. However, during the ICME storm 

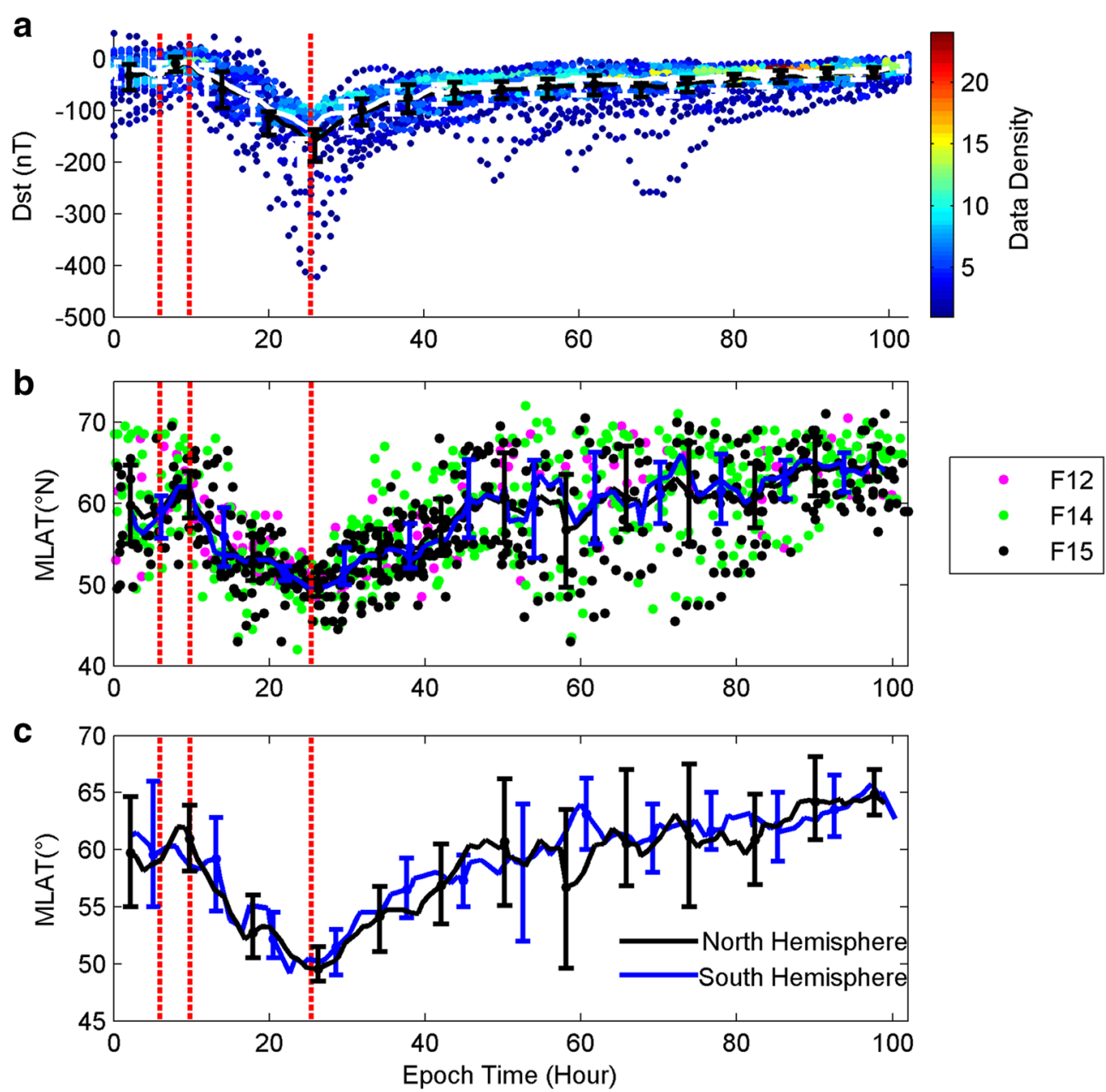

Fig. 3 Superposed epoch plots of the Dst index (a), Northern Hemisphere trough positions (b) and the average trough positions in both hemispheres (c) for $33 \mathrm{MC}$ storms. The color of the plot in a presents the density of data points in $5 \mathrm{nT}$ by 3-h bins. The black and white lines in a indicate the mean and the median, respectively. The pink, green and black circles in b indicate the data derived from DMSP-F12, DMSP-F14 and DMSP-F15, respectively. The black and blue lines in $\mathbf{b}$ indicate the mean and the median, respectively. The black and blue lines in $\mathbf{c}$ indicate the results of the Northern Hemisphere and Southern Hemisphere, respectively. The short vertical lines in each figure indicate the range bounded by the upper and lower quartiles. The red vertical lines show the start or end of each phase

recovery phase, changes in the trough position are consistent with those in the Dst index.

\section{Superposed epoch analysis results for moderate storms and intense storms}

Figure 5 shows the statistic results in the same format as Fig. 4, but for moderate (a) and intense (b) storms. The numbers of moderate and intense storms are 41 and 46 , respectively. It is evident that intense storms have longer durations, both for the main phase and for the recovery phase. The trough position is closer to the equator at the end of the intense storm main phase. During the storm recovery phase, the trough position recovers rapidly at the start of the moderate storm recovery phase, but this feature disappears at the start of the intense storm recovery phase. In addition, this feature is also pronounced during the CIR storm recovery phase (see Fig. 4a). Therefore, this feature is associated with moderate CIR storms. This finding is verified through the analysis of the trough position for moderate CIR storms.

\section{Superposed epoch analysis results for different solar wind drivers}

Figure 6 shows the results of the superposed epoch analysis for the four categories of magnetic storms with the peak Dst between -100 and -150 nT. Figure 2c presents the fact that the variations in the Dst index during the storm recovery phase can result in large variations 

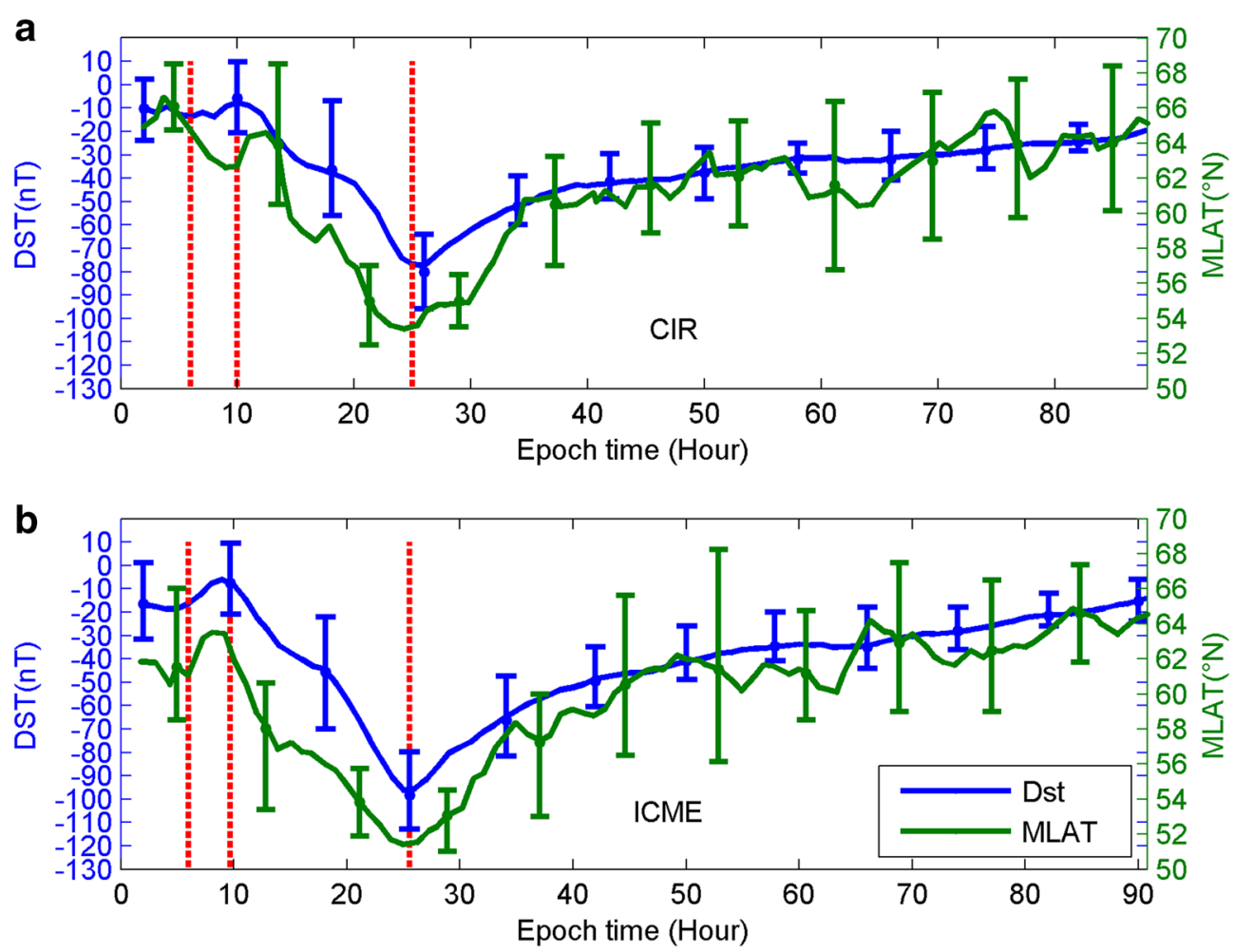

Fig. 4 Superposed epoch analysis of Dst index and trough position for CIR (a) and ICME (b) storms using the normalized time line. The solid blue lines indicate the average Dst index. The solid green lines indicate the average trough position. The short vertical lines indicate the range bounded by the upper and lower quartiles. The red vertical lines show the start or end of each phase

in the trough position. Therefore, the storms with large variations in the Dst index during the recovery phase are removed. Table 1 shows several parameters for the four categories of magnetic storms. It is evident that these plots are quite similar to each other. The trough moves toward the equator as Dst index decreases and toward the pole as Dst index increases. For CIR, ICME and MC storms, the minimum latitude of the trough position decreases as the storm magnitude increases. The minimum latitude of the trough position for sheath storms is closer to the equator, although the average storm intensity is weak. In addition, it is striking that the average duration of the main phase is shortest for sheath storms. It seems that the minimum latitude of the trough position correlates with the duration of the main phase, or the rate of Dst decreases during the main phase, or the magnitude of Dst decreases during the main phase.

Figure 7 shows the relationship between the minimum latitude of the trough position and the storm magnitude. The data in Fig. 7a, b are derived from DMSP and DEMETER satellites, respectively. The correlation coefficients and $P$ values between the minimum latitude of the trough position and the Dst index at the start of main phase (Dstmax, second row), the magnitude of Dst decrease during the main phase ( $\Delta$ Dst, third row), the duration of main phase $(\Delta T$, forth row), the rate of Dst decrease during the main phase $(\Delta \mathrm{Dst} / \Delta \mathrm{T}$, fifth row) and the Dst peak value (Dstmin, sixth row) are summarized in Table 2 . It is evident that there is a strong (correlation coefficients $>0.8)$ and significant $(P$ value $<0.0001)$ correlation between the minimum latitude of the trough position and the Dstmin. Because $\Delta$ Dst correlates with Dstmin, the correlation with $P$ value $<0.001$ between the minimum latitude of the trough position and $\Delta$ Dst are also statistically significant. However, the correlation between the minimum latitude of the trough position and Dstmax and $\Delta \mathrm{T}$ is weak (correlation coefficient $<0.3$ ) and not significant ( $P$ value $>0.1$ ). So, the minimum latitude of the trough position does not correlate with other parameters associated with the main phase. The minimum latitude of the trough position is largely dependent on Dstmin for all storms. As the Dstmin decreases, the trough moves to lower latitude.

\section{Discussion}

In this paper, we specify the statistical characteristics of the trough position for different categories of magnetic storms and different storm intensities. During magnetic 

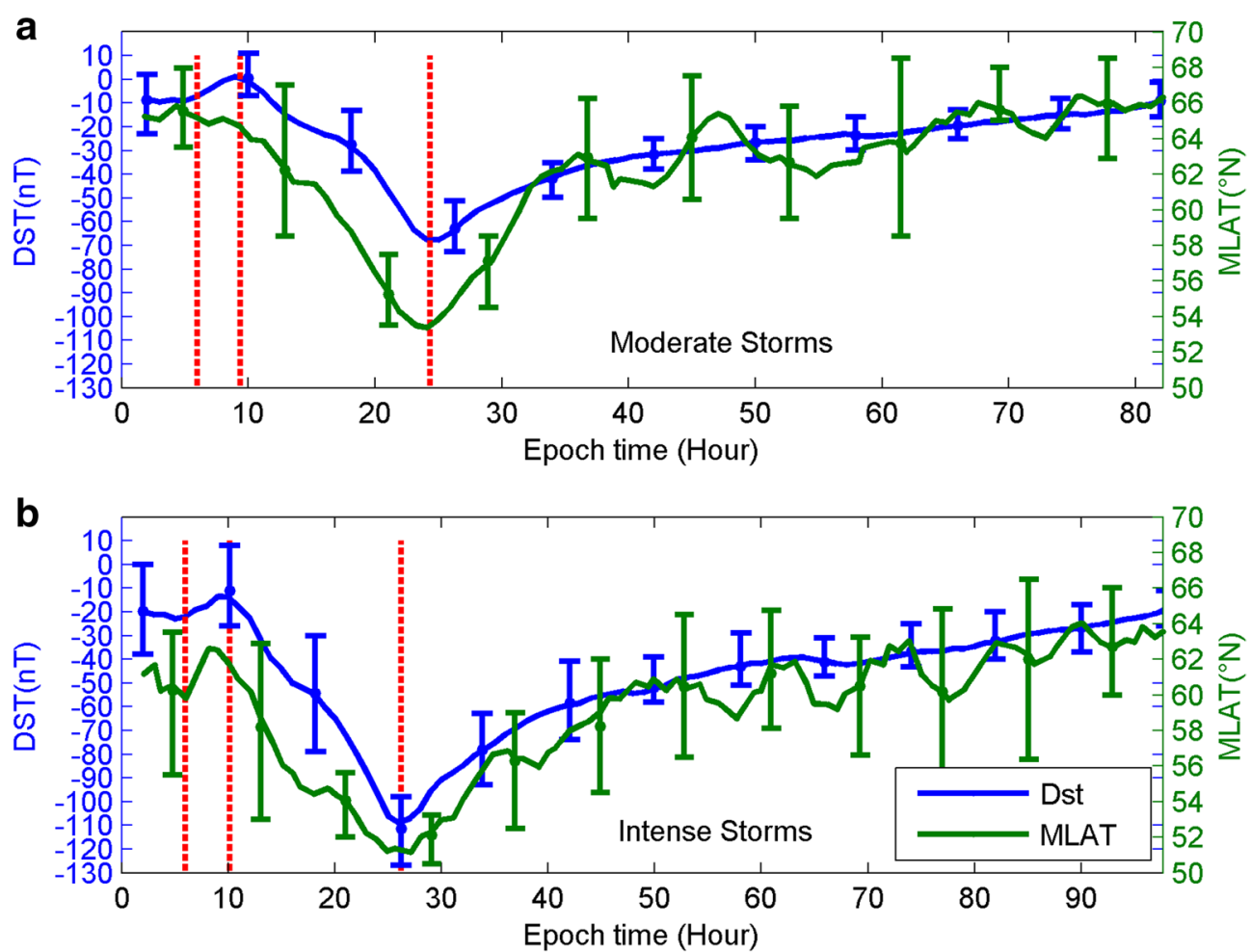

Fig. 5 Superposed epoch analysis of Dst index and trough position for moderate (a) and intense (b) storms using the normalized time line. The solid blue lines indicate the average Dst index. The solid green lines indicate the average trough position. The short vertical lines indicate the range bounded by the upper and lower quartiles. The red vertical lines show the start or end of each phase

storms, the longitudinal effect in the trough position is weak and can be neglected. The result is consistent with the finding of Karpachev et al. (1996). In addition, the temporal evolution of the trough position in the Northern and Southern Hemispheres during magnetic storms is symmetric.

During the main phase of magnetic storms, the average trough position moves equatorward with decreasing Dst index. During this phase, an increase in the intensity of the ring current after the rotation of the vertical component of interplanetary magnetic field (IMF) Bz from north to south is accompanied by the displacement of the inner boundary of plasma sheet ions toward the Earth (Hamilton et al. 1988). Since this boundary is connected with the trough, the trough position follows the variation and moves equatorward (Deminov et al. 1995a). Compared with the ICME, MC and CIR storms, in sheath storms the trough shifts to lower latitude at the end of the main phase. This phenomenon is associated with the solar wind properties of sheath storms. Previous studies have addressed the similarities and differences of the dominant solar wind parameters for the four categories of magnetic storms with the peak Dst between -100 and $-150 \mathrm{nT}$ (Katus et al. 2015b). During the main phase of sheath storms, the greater inner magnetospheric convection associated with larger southward IMF Bz and solar wind velocity exists. The greater inner magnetospheric convection can result in the larger displacement of the inner boundary of plasma sheet and the trough position. In addition, it has also been demonstrated in our study that the minimum latitude of the trough position is largely dependent on the magnitude of the storms. The minimum latitude of the trough position does not correlate with other parameters associated with the main phase.

During the recovery phase of magnetic storms, the average trough position moves poleward with increasing Dst index. The timescale for recovery of the trough is of the order of hours (see Fig. 2c). It is striking that the rapid recovery of the trough position occurs at the start of the recovery phase for moderate CIR storms. For strong CIR storms and ICME storms, changes in the trough position follow Dst index variations. To understand the cause of the differences, this study examines the dominant solar wind parameters for moderate and intense CIR storms. Figure 8 shows superposed epoch solar wind parameters (solar wind electric field, solar wind dynamic pressure and IMF Bz) for moderate (left panels) and intense 

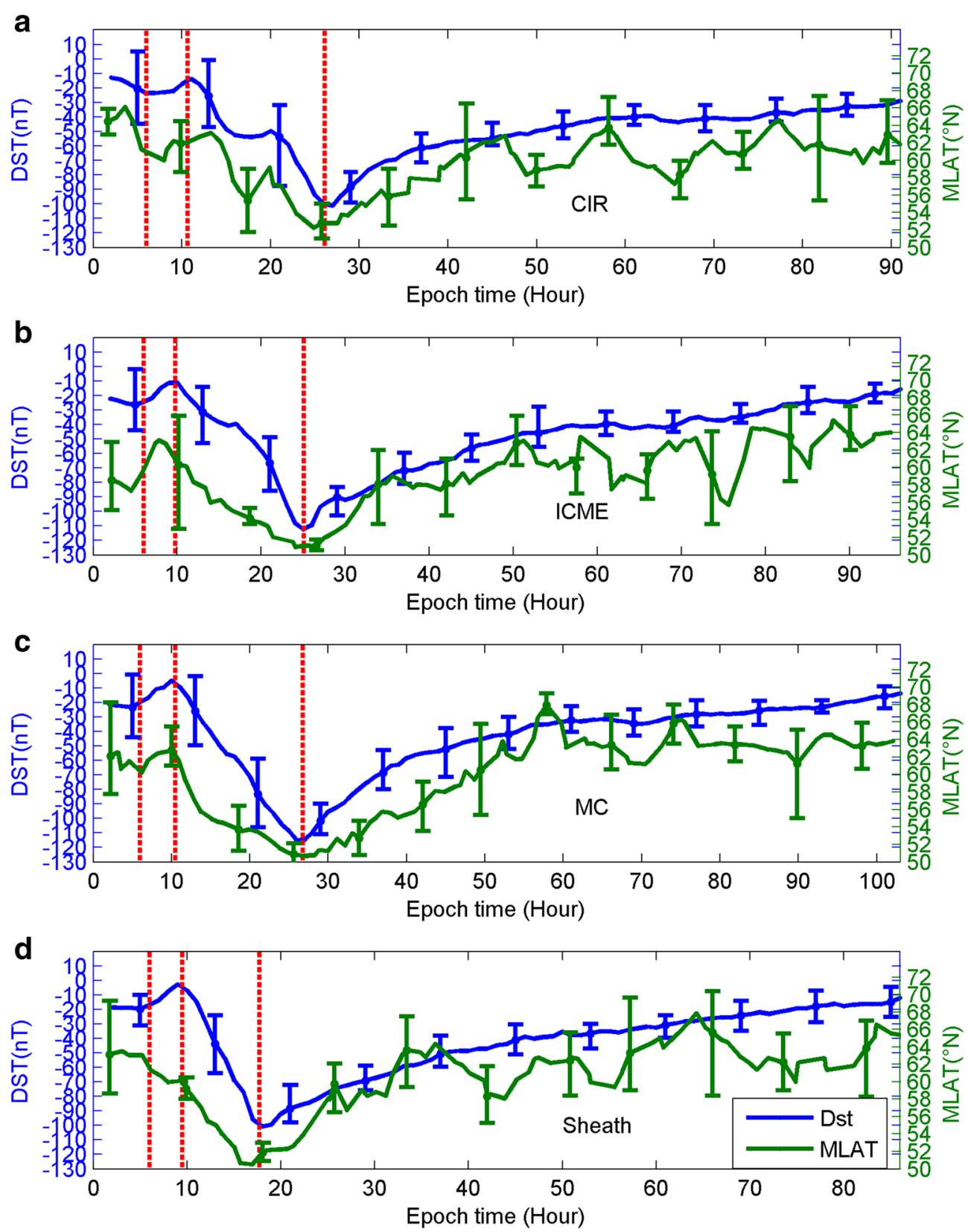

Fig. 6 Superposed epoch analysis of Dst index and trough position for the four categories of magnetic storms using the normalized time line. The solid blue lines indicate the average Dst index. The solid green lines indicate the average trough position. The short vertical lines indicate the range bounded by the upper and lower quartiles. The red vertical lines show the start or end of each phase

(right panels) CIR storms. These hourly averaged data are taken from the high-time-resolution (1 min) OMNI database (http://omniweb.gsfc.nasa.gov/). The red vertical lines show the start or end of each phase. It is striking that these parameters continue to vary at the start of the recovery phase for moderate storms, but remain constant for intense storms. This phenomenon is not found at the start of the ICME storm recovery phase. These differences of solar wind parameters may lead to the rapid recovery of the trough position for moderate CIR storms.

Although theoretically CIRs are essentially sheaths, their solar wind properties are very different. These differences may lead to different behaviors of the trough. Therefore, future work should be carried out to examine the relationship between the various solar wind parameters and the trough. 
Table 1 Parameters characterizing the four categories of magnetic storms

\begin{tabular}{lccrc}
\hline & CIR (11) & ICME (14) & MC (18) & Sheath (10) \\
\hline Main phase duration (h) & 15.45 & 15.29 & 16.28 & 8.2 \\
Recovery phase duration & 67.9 & 73.07 & 79.11 & 71.1 \\
(h) & & & & \\
Dst peak index (nT) & -100.1 & -112.3 & -114.6 & -100.8 \\
Trough peak position (N) & 52.22 & 50.89 & 50.69 & 50.5 \\
\hline
\end{tabular}

In brackets, the number of storms is shown

\section{Conclusions}

This study shows evidence that the trough dynamics investigated are different for different categories of magnetic storms and different storm intensities. The properties of trough position during magnetic storms may be summarized as follows.

(1) The temporal evolution of the trough position in the Northern and Southern Hemispheres during magnetic storms is symmetric.

(2) During the main phase of magnetic storms, the average trough position moves equatorward with decreasing Dst index. Compared with the ICME, $\mathrm{MC}$ and CIR storms, in sheath storms the trough shifts to lower latitude at the end of the main phase, although the average storm intensity is weak.

(3) During the recovery phase of magnetic storms, the average trough position moves poleward with
Table 2 Correlation coefficients (cc) and $P$ values for different satellite data

\begin{tabular}{|c|c|c|c|c|}
\hline & \multicolumn{2}{|c|}{ DMSP (31) } & \multicolumn{2}{|c|}{ DEMETER (23) } \\
\hline & Cc & $P$ value & Cc & $P$ value \\
\hline Trough and Dst ${ }_{\max }$ & 0.38 & 0.11 & 0.04 & 0.86 \\
\hline Trough and $\Delta$ Dst & -0.59 & 0.0007 & -0.79 & $<0.0001$ \\
\hline Trough and $\Delta \mathrm{T}$ & 0.19 & 0.24 & 0.17 & 0.44 \\
\hline Trough and $\triangle D s t / \Delta T$ & -0.45 & 0.01 & -0.62 & 0.001 \\
\hline Trough and Dst $t_{\min }$ & 0.81 & $<0.0001$ & 0.86 & $<0.0001$ \\
\hline
\end{tabular}

increasing Dst index. The rapid recovery of the trough position can be seen at the start of the recovery phase for moderate CIR storms.

(4) As the magnitude of magnetic storm increases, the trough moves to lower latitude.

As a conclusion, during the sheath and CIR storms, the trough's behavior is different from that during the ICME and MC storms. During the ICME and MC storms, changes in the trough position are consistent with those in the Dst index. Compared with the ICME, MC and CIR storms, in sheath storms the trough shifts to lower latitude at the end of the main phase, although the average storm intensity is weak. The rapid recovery of the trough position appears at the start of the recovery phase in the moderate CIR storms but not in ICME and MC storms.
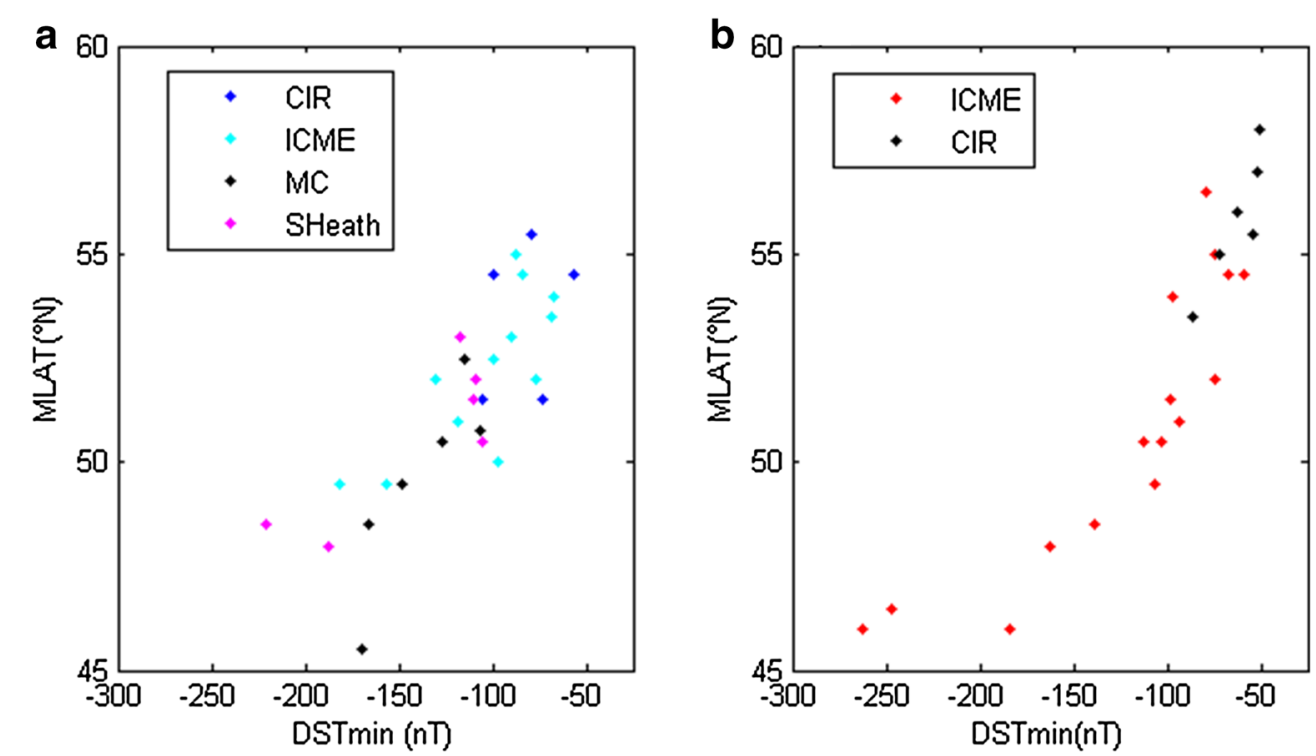

Fig. 7 Relationship between the minimum latitude of the trough position and the storm magnitude. The data in $\mathbf{a}$ and $\mathbf{b}$ are derived from DMSP and DEMETER satellites, respectively 

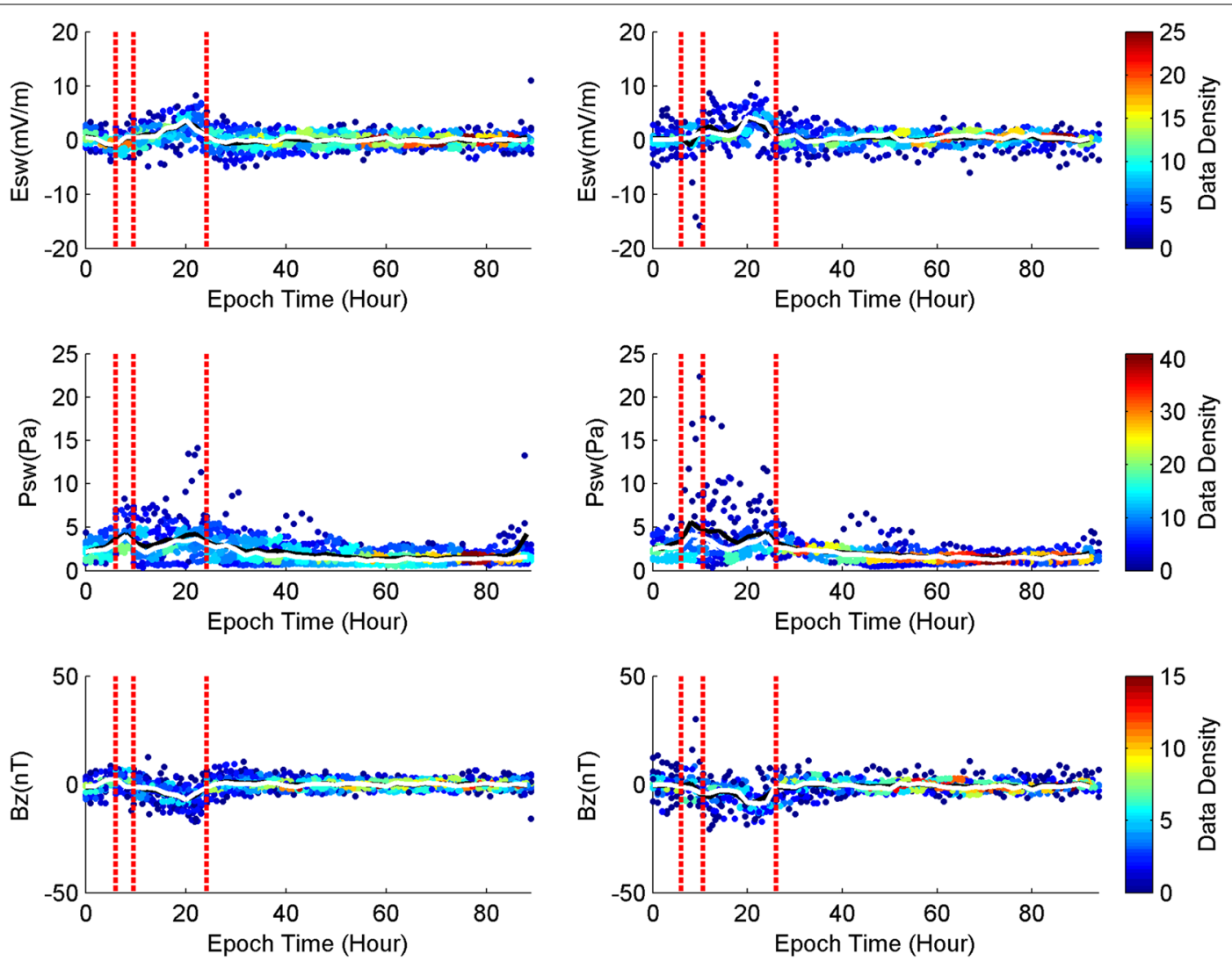

Fig. 8 Superposed epoch plots of solar wind parameters (solar wind electric field, solar wind dynamic pressure and IMF Bz) for moderate (left panels) and intense (right panels) CIR storms. The black and white lines indicate the mean and the median, respectively. The red vertical lines show the start or end of each phase

This information is likely to assist in validating and testing ionospheric models where the trough structure is included.

\section{Authors' contributions}

NY and HL planned and led the study, interpreted the results and drafted the manuscript. LL participated in the data analysis and interpretation. All authors read and approved the final manuscript.

\section{Author details}

${ }^{1}$ Key Laboratory of Earth and Planetary Physics, Institute of Geology and Geophysics, Chinese Academy of Sciences, Beijing, China. ${ }^{2}$ Beijing National Observatory of Space Environment, Institute of Geology and Geophysics, Chinese Academy of Sciences, Beijing, China. ${ }^{3}$ University of the Chinese Academy of Sciences, Beijing 100049, China.

\section{Acknowledgements}

This research was supported by the National Key Basic Research Program of China (2012CB825604), the National Natural Science Foundation of China (41231065, 41374162 and 41321003) and Youth Innovation Promotion Association CAS

\section{Competing interests}

The authors declare that they have no competing interests.

Received: 12 May 2016 Accepted: 26 October 2016

Published online: 04 November 2016

\section{References}

Borovsky JE, Denton MH (2006) Differences between CME-driven storms and CIR-driven storms. J Geophys Res 111:A07S08. doi:10.1029/2005JA011447

Burlaga L, Sittler E, Mariani F, Schwenn R (1981) Magnetic loop behind an interplanetary shock: Voyager, Helios and IMP 8 observations. J Geophys Res 86:6673-6684

Deminov MG, Karpachev AT, Afonin VV, Annakuliev SK, Ya Shmilauer (1995a) Dynamics of mid-latitude ionospheric trough during the storm. I. Qualitative pattern. Geomagn Aeron 35(1):73-79

Deminov MG, Karpachev AT, Afonin VV, Annakuliev SK (1995b) Dynamics of mid-latitude trough during the magnetic storm: main phase. Geomagn Aeron 35(6):69-77

Deminov MG, Karpachev AT, Annakuliev SK, Afonin VV, Ya Shmilauer (1996) Dynamics of the ionization troughs in the nighttime subauroral F-region during geomagnetic storms. Adv Space Res 17(10):141-145

Denton MH, Borovsky JE, Skoug RM, Thomsen MF, Lavraud B, Henderson MG, McPherron RL, Zhang JC, Liemohn MW (2006) Geomagnetic storms driven by ICME- and CIR-dominated solar wind. J Geophys Res 111:A07S07. doi:10.1029/2005JA011436

Dmitriev AV, Crosby NB, Chao JK (2005) Interplanetary sources of space weather disturbances in 1997 to 2000. Space Weather 3:S03001. doi:10.1 029/2004SW000104

Hamilton D, Gloeckler G, Iravich FM, Stüdemann W, Wilken B, Kremser G (1988) Ring current development during the great geomagnetic storm of February 1986. J Geophys Res 93(A12):14343-14355

He M, Liu L, Wan W, Zhao B (2011) A study on the nighttime midlatitude ionospheric trough. J Geophys Res 116:A05315. doi:10.1029/2010JA016252 
Horvath I, Essex EA (2003) The southern-hemisphere mid-latitude day-time and nighttime trough at low-sunspot numbers. J Atmos Terr Phys 65:917-940

Huttunen KEJ, Koskinen HEJ, Schwenn R (2002) Variability of magnetospheric storms driven by different solar wind perturbations. J Geophys Res 107(A7):1121. doi:10.1029/2001JA900171

Ishida T, Ogawa Y, Kadokura A, Hiraki Y, Häggström I (2014) Seasonal variation and solar activity dependence of the quiet-time ionospheric trough. J Geophys Res Space Phys. doi:10.1002/2014JA019996

Jian L, Russell CT, Luhmann JG, Skoug RM (2006) Properties of stream interactions at one AU during 1995-2004. Sol Phys 239:337-392. doi:10.1007/ s11207-006-0132-3

Karpachev AT, Deminov MG, Afonin WV (1996) Model of the mid-latitude ionospheric trough on the base of cosmos-900 and intercosmos-19 satellites data. Adv Space Res 18:6221-6230

Katus RM, Liemohn MW, Gallagher DL, Ridley A, Zou S (2013) Evidence for potential and inductive convection during intense geomagnetic events using normalized superposed epoch analysis. J Geophys Res Space Phys 118:181-191. doi:10.1029/2012JA017915

Katus RM, Liemohn MW, lonides EL, llie R, Welling D, Sarno-Smith LK (2015a) Statistic al analysis of the geomagnetic response to different solar wind drivers and the dependence on storm intensity. J Geophys Res Space Phys 120:310-327. doi:10.1002/2014JA020712

Katus RM, Gallagher DL, Liemohn MW, Keesee AM, Sarno-Smith LK (2015b) Statistical storm time examination of MLT-dependent plasmapause location derived from IMAGE EUV. J Geophys Res Space Phys. doi:10.1002/20 15JA021225

Klein LW, Burlaga LF (1982) Interplanetary magnetic clouds at 1 AU. J Geophys Res 87:613-624

Krieger AS, Timothy AF, Roelof EC (1973) A coronal hole and it's identification as the source of a high velocity solar wind stream. Sol Phys 29:505-525. doi:10.1007/BF00150828

Lee I, Liu JY, Wang W, Chen C, Lin C (2011) The ionospheric mid-latitude trough observed by FORMOSAT-3/COSMIC during solar minimum. J Geophys Res 116:A06311. doi:10.1029/2010JA015544

Middleton HR, Pryse SE, Wood AG, Balthazor R (2008) The role of the tongueof-ionization in the formation of the poleward wall of the main trough in the European post-midnight sector. J Geophys Res 113:A02306. doi:10.10 29/2007JA012631

Muldrew DB (1965) F-layer ionization troughs deduced from Alouette data. J Geophys Res 70(11):2635-2650

Nilsson H, Sergienko TI, Ebihara Y, Yamauchi M (2005) Quiet-time mid-latitude trough: influence of convection field-aligned currents and proton precipitation. Ann Geophys 23:3277-3288

Pryse SE, Kersley L, Malan D, Bishop GJ (2006) Parameterization of the main ionospheric trough in the European sector. Radio Sci 41:RS5S14. doi:10.1 029/2005RS003364
Richardson IG, Cane HV (2010) Near-Earth interplanetary coronal mass ejections during solar cycle 23 (1996-2009): catalog and summary of properties. Sol Phys 264:189-237. doi:10.1007/s11207-010-9568-6

Richardson IG, Cane HV (2012) Near-earth solar windows and related geomagnetic activity during more than four solar cycles (1963-2011). J Space Weather Space Clim 2:A02. doi:10.1051/swsc/2012003

Rodger A (2008) The mid-latitude trough: revisited. In: Midlatitude ionospheric dynamics and disturbances. Geophys Monogr Ser, vol 181. AGU, Washington, DC, pp 25-33

Rodger AS, Dudeney JR (1987) Is the poleward edge of the trough a sensitive indicator of geospace interactions? Adv Space Res 7(8):65-74. doi:10.1016/0273-1177(87)90191-8

Rodger AS, Brace LH, Hoegy WR, Winningham JD (1986) The poleward edge of the mid-latitude trough-its formation, orientation and dynamics. J Atmos Sol Terr Phys 48(8):715-728

Rodger AS, Moffett RJ, Quegan S (1992) The role of ion drift in the formation of ionisation troughs in the mid-and high-latitude ionosphere-a review. J Atmos Sol Terr Phys 54(1):1-30

Scali IL (1992) The mid-latitude trough: a review. Cent for Atmos Res Mass Univ Lowell, Lowell

Tsurutani BT, Gonzalez WD, Gonzalez AL, Guarnieri FL, Gopalswamy N, Grande M, Kamide Y, Kasahara Y, Lu G, Mann I, McPherron R, Soraas F, Vasyliunas V (2006) Corotating solar wind streams and recurrent geomagnetic activity: a review. J Geophys Res Space Phys 111(A7):a07S01. doi:10.1029/200 5JA011273

Webb DF, Howard TA (2012) Coronal mass ejections: observations. Living Rev Solar Phys. doi:10.1007//rsp-2012-3

Wilken B, Zong QG, Reeves GD, Doke T, Yamamoto T (1999) Geoactivity in response to CIR/CME events - a synoptic view. Phys Chem Earth C 24(1):113-117

Yang N, Le H, Liu L (2015) Statistical analysis of ionospheric mid-latitude trough over the Northern Hemisphere derived from GPS total electron content data. Earth Planets Space 67(1):1-11. doi:10.1186/s40623-015-0365-1

Zhang J, Richardson IG, Webb DF, Gopalswamy N, Huttunen E, Kasper JC, Nitta NV, Poomvises W, Thompson BJ, Wu CC, Yashiro S (2007a) Solar and interplanetary sources of major geomagnetic storms (Dst $\leq-100 \mathrm{nT}$ ) during 1996-2005. J Geophys Res Space Phys 112:A10102. doi:10.1029/2 007JA012321

Zhang J, Richardson IG, Webb DF, Gopalswamy N, Huttunen E, Kasper JC, Nitta NV, Poomvises W, Thompson BJ, Wu CC, Yashiro S, Zhukov AN (2007b) Correction to "Solar and interplanetary sources of major geomagnetic storms (Dst $\leq 100$ nT) during 1996-2005". J Geophys Res 112:A12103. doi :10.1029/2007JA012891

Zou S, Moldwin MB, Coster A, Lyons LR, Nicolls MJ (2011) GPS TEC observations of dynamics of the mid-latitude trough during substorms. Geophys Res Lett 38:L14109. doi:10.1029/2011GL048178

\section{Submit your manuscript to a SpringerOpen ${ }^{\circ}$ journal and benefit from:}

- Convenient online submission

- Rigorous peer review

- Immediate publication on acceptance

- Open access: articles freely available online

- High visibility within the field

- Retaining the copyright to your article

Submit your next manuscript at $\boldsymbol{\nabla}$ springeropen.com 